\title{
Nucleation Parameters of SPC/E And TIP4P/2005 Water Vapor Measured In NPT Molecular Dynamics Simulations
}

\section{Tomáš Němec ( $\square$ nemec@it.cas.cz )}

Institute of Thermomechanics of the Czech Academy of Sciences https://orcid.org/0000-0002-3456$933 \mathrm{X}$

\section{Research Article}

Keywords: water nucleation, molecular dynamics, SPC/E, TIP4P/2005

Posted Date: January 3rd, 2022

DOI: https://doi.org/10.21203/rs.3.rs-1177344/v1

License: (c) (1) This work is licensed under a Creative Commons Attribution 4.0 International License.

Read Full License 


\title{
Nucleation parameters of SPC/E and TIP4P/2005 water vapor measured in NPT molecular dynamics simulations
}

\author{
Tomáš Němec ${ }^{1 *}$ \\ $1^{*}$ Department of Electrical Engineering and Electrophysics, \\ Institute of Thermomechanics of the Czech Academy of Sciences, \\ v. v. i., Dolejškova 5, Praha 8, 18200, Czech Republic.
}

Corresponding author(s).E-mail(s):nemec@it.cas.cz;

\begin{abstract}
Nucleation rates for droplet formation in water vapor are measured in molecular dynamics simulations of SPC/E and TIP4P/2005 water by monitoring individual nucleation events. The nucleation process is simulated in the NPT ensemble to evaluate the steady-state nucleation rate in accordance with the assumptions of classical nucleation theory (CNT). Nucleation rates measured between $300 \mathrm{~K}$ and $425 \mathrm{~K}$ for the SPC/E model, and between $325 \mathrm{~K}$ and $475 \mathrm{~K}$ for the TIP4P/2005 model, agree with the CNT predictions roughly within the standard deviation of the MD measurements of the nucleation rates.
\end{abstract}

Keywords: water nucleation, molecular dynamics, SPC/E, TIP4P/2005

\section{Introduction}

The aim of this work is to observe steady-state nucleation events in numerical molecular dynamics (MD) experiments and to evaluate nucleation parameters for droplet nucleation in water vapor. Two water models, SPC/E [1] and TIP4P/2005 [2], are used in this work. These two models have been subjected to several nucleation studies in the past and reference nucleation rate data can be found in the literature for SPC/E [3-7] and also for TIP4P/2005 [811]. Before the introduction of the TIP $4 \mathrm{P} / 2005$ model, an important study 
on nucleation using the TIP4P model [12] appeared, in which the YasuokaMatsumoto method for evaluation of nucleation rate from MD simulations was introduced. This method was used in the majority of subsequent MD nucleation studies.

Existing MD water nucleation studies differ in the amount of nucleation rate data reported. Simple studies include only one simulation run [9, 11], while comprehensive numerical work [7] has collected nucleation rate data over a wide range of temperatures and supersaturations. Accordingly, the computational resources used in the various studies vary by many orders of magnitude. For example, Dumitrescu et al. [10] report 12 simulation runs, each of which consumed about 2.6 thousand CPU hours. On the other hand, Angelil et al. [7] report 18 large-scale simulation runs, the largest of which consumed 8.2 million CPU hours.

An often-discussed feature of the experimental nucleation data is their difference from the theoretical predictions of classical nucleation theory (CNT). In previous works, CNT predictions of the nucleation rate typically differ by many orders of magnitude from the experimental results, which is the case for both MD nucleation simulations of various water models and physical nucleation experiments in laboratory [13]. For nucleation data listed in Table 1 from MD, variations of one to three orders of magnitude are typical for the nucleation rate. The critical cluster size is underestimated by a factor between 2 and $45 \times$. This is similar to the situation with physical nucleation experiments in water vapor, where discrepancies in the nucleation rate have already been quantified in terms of the so-called Wölk correction function [14].

In order to perform a thorough comparison between the MD nucleation rates and the CNT predictions, attention must be paid to ensuring that the nucleation processes are compatible with the assumptions of the CNT statistical-mechanical model. Since the CNT model describes a steady-state nucleation process, i.e. a process of cluster formation in which the thermodynamic state of the parent vapor phase is not affected by the cluster formation, the same conditions must be ensured in the corresponding MD simulations. In the terminology of MD simulations, such steady-state nucleation processes are realized as NPT processes, where the temperature and pressure are kept constant. Therefore, NPT simulations will be considered in this work, which is the main difference from the previous MD studies of droplet nucleation in water vapor, mostly performed in NVT, but also in NVE ensembles.

The most often used methods for the evaluation of nucleation rate in MD simulations can be characterized as indirect. Typically, evolution of cluster size distribution is being observed during the course of the simulation and the distribution is linked to the nucleation rate by relations originating from nucleation theory equations. In the established Yasuoka-Matsumoto method[12], the nucleation rate is evaluated according to the slopes of number of clusters of a given size as a function of simulation time. Another indirect method for nucleation rate evaluation, the mean-first passage time (MFPT) method [8], measures first apearances in time of certain-sized clusters in the simulation and 
Table 1 Summary of MD nucleation studies in SPC/E and TIP4P/2005 water models. Differences between MD nucleation parameters and CNT predictions are shown for nucleation rate $J$ and critical cluster size $n^{\star}$ as reported by the respective authors.

\begin{tabular}{lcccc}
\hline & Water model & Temperature [K] & $J_{M D} / J_{C N T}$ & $n_{M D}^{\star} / n_{C N T}^{\star}$ \\
\hline Matsubara (2007) [3] & SPC/E & $275-375$ & $10^{-2}-10^{-1}(a)$ & $3-30(a)$ \\
Sepehri (2013) [4] & SPC/E & 350 & 5.6 & $2.3-2.6$ \\
Tanaka (2014) [6] & SPC/E & $250-375$ & $10^{-1}$ & $1.4-5$ \\
Zhang (2015) [5] & SPC/E & 370,390 & $0.71,0.3$ & $2.6,1.8$ \\
Angélil (2015) [7] & SPC/E & $300-375$ & $10^{-2}-10^{-1}$ & $30-45$ \\
\hline Yasuoka (1998) [12] & TIP4P & 350 & $10^{-3}$ & $3.5-4$ \\
Perez (2011) [8] & TIP4P/2005 & 330 & $10^{3}$ & 2 \\
Duška (2015) [9] & TIP4P/2005 & 347.3 & $10^{-1}$ & $\sim 1$ \\
Dumitrescu (2017) [10] & TIP4P/2005 & 350 & $0.35-0.7(b)$ & \\
Hrubý (2018) [11] & TIP4P/2005 & 419.8 & & \\
\hline
\end{tabular}

(a) values estimated from figures

(b) single-run NVE simulation

links the first passages to an error-function-like dependency based on nucleation theory relations. A high number of simulation runs (several hundreds) are being performed to gather reliable statistics for MFPT evaluation.

The approach presented in this work for nucleation rate evaluation lies in a direct observation of nucleation events, i.e. no additional theoretical considerations need to be invoked. Each simulation run is set up to represent a single nucleation event, which is observed after a well defined period of subcritical cluster size fluctuations in the metastable parent phase, followed by the growth of a supercritical droplet. These two simulation stages need to be clearly distinguishable within each simulation run as the transition between these two stages corresponds to the event of nucleation. The setup of our numerical experiments is described in Sec. 2 together with the numerical evaluation of $\mathrm{MD}$ nucleation parameters, i.e. nucleation rate, impingement rate, critical cluster size and critical nucleation work. The agreement between nucleation parameters from MD simulations and CNT predictions is discussed in Sec. 3.

\section{Numerical experiments}

Numerical experiments reported in this work have been performed by using the DL_POLY 4.08 molecular dynamics software [15]. For both water models, SPC/E and TIP4P/2005, a cut-off radius of $13 \AA$ was used. Ewald precision parameter for electrostatics was $10^{6}$. The cut-off radius was chosen to comply with MD simulations performed to evaluate thermophysical properties of SPC/E and TIP4P/2005 water models (Appendix D and E).

Each numerical experiment consists of the following steps:

A. Prediction of system pressure at target temperature

B. NVT system equilibration at elevated temperature

C. NPT evolution of the metastable system at target temperature and pressure 
The last step $\mathrm{C}$ is further divided into two stages:

1. Incubation stage of the metastable vapor

2. Growth of the supercritical liquid droplet

The transition between stages 1 and 2 will be considered as the nucleation event, and the time instant when this transition appears will be referred to as the nucleation time.

The first step is to estimate the initial parameters of the simulated system. For a target temperature, we need to estimate the pressure of the system at which a nucleation event can be observed in a reasonable time frame. On the one hand, the nucleation time must fall within a realistic time interval that can be achieved by the numerical simulation, and on the other hand, the nucleation time must be long enough to allow a clear distinction between an incubation stage and a growth stage of the nucleation process. Nucleation times of about 10 ns meet these requirements and were therefore targeted in our simulations.

Each simulation run represents a single nucleation event. For each thermodynamic state, characterized by its temperature and pressure, a total of 20 simulation runs were performed, each starting from different initial positions of water molecules, in order to collect reliable statistics for the evaluation of the average nucleation rate and its standard deviation.

Molecular systems of three sizes were simulated, i.e. 108, 500, and 2916 molecules, which resulted in nucleation rates in the range between $10^{30}$ and $10^{33} \mathrm{~m}^{-3} \mathrm{~s}^{-1}$. The details of the simulation procedure are described in the following paragraphs. To illustrate our thought process in evaluating the nucleation rate using molecular simulations, an example experiment MD was selected and described in detail. The example system considered in the following paragraphs features $500 \mathrm{TIP} 4 \mathrm{P} / 2005$ molecules at $425 \mathrm{~K}$ and $590 \mathrm{kPa}$.

\subsection{Classical nucleation theory predictions}

We use CNT as a reference for the nucleation rate measurements in molecular simulations. For a given temperature $T$, CNT is used to find pressure $p$ at which a desired nucleation rate should be observed. The CNT nucleation rate $J_{C N T}$ then represents a reference value for the nucleation rate $J_{M D}$ measured by molecular simulations. We use the standard form of CNT for unary homogeneous droplet nucleation as summarized in Appendix A. CNT is employed in its basic form without empirical corrections introduced in the past for specific purposes in the nucleation literature. The thermophysical properties of the water models required by CNT are summarized in Appendix D and E, for the SPC/E model and the TIP4P/2005 model, respectively.

The nucleation parameters of our example system as predicted by CNT are listed in Table 2. This tells us that our system with 500 TIP $4 \mathrm{P} / 2005$ molecules, if allowed to evolve in an NPT simulation at $425 \mathrm{~K}$ and $590 \mathrm{kPa}$, is expected to nucleate one liquid drop after $9.5 \mathrm{~ns}$ on average. This corresponds to a steady-state nucleation rate of $2.2 \times 10^{33} \mathrm{~m}^{-3} \mathrm{~s}^{-1}$. The technical details of the molecular simulation run are described below. 
Table 2 CNT prediction of nucleation parameters for 500 TIP4P/2005 molecules at $425 \mathrm{~K}$ and $590 \mathrm{kPa}$. The corresponding supersaturation is 2.68 .

\begin{tabular}{llll}
\hline critical cluster & $n^{\star}$ & 19.5 & \\
nucleation rate & $J$ & $2.2 \times 10^{31}$ & $\mathrm{~m}^{-3} \mathrm{~s}^{-1}$ \\
impingement rate & $\beta$ & $1.8 \times 10^{28}$ & $\mathrm{~m}^{-2} \mathrm{~s}^{-1}$ \\
\hline system density & $\rho_{M D}$ & $1.04 \times 10^{-4}$ & $\AA^{-3}$ \\
box size & $l$ & 168.98 & $\AA$ \\
nucleation time & $t_{n u c}$ & 9.5 & $\mathrm{~ns}$ \\
\hline
\end{tabular}

\subsection{System equilibration at elevated temperature}

An equilibration NVT run at $1000 \mathrm{~K}$ is performed at first in order to generate a pool of initial configurations for subsequent NPT nucleation simulation runs. The reason for performing the equilibration run at supercritical temperature is to prevent the formation of larger clusters during this initial simulation stage. Andersen thermostat was used with 0.5 and 0.5 relaxation constants to control the temperature in this step.

The volume of the NVT system at elevated temperature is adjusted so that the density of the system corresponds to the intended water vapor density at the target simulation temperature and pressure, as given in Table 2 for our example case. This is accomplished by multiplying the saturation density of water vapor at the target simulation temperature by the target supersaturation, which gives us the system density.

The NVT equilibration is performed for $5 \mathrm{~ns}$ with a time step of $1 \mathrm{fs}$, storing the configuration history every 500 time steps. The generated configurations, i.e. atomic positions and velocities, in the period between $1 \mathrm{~ns}$ and $5 \mathrm{~ns}$ of the equilibration run are used as a pool for randomly selecting 20 initial configurations to be used in the next simulation step.

\subsection{Metastable vapor}

Each configuration generated at $1000 \mathrm{~K}$ is used as the initial configuration in a new NPT simulation run by scaling the velocities to the target simulation temperature (using the restart scale directive of DL_POLY). Since the volume of the system was adjusted in the previous equilibration run to match the density of the water vapor at the target temperature and pressure, a relatively smooth transition from the NVT ensemble to the NPT ensemble is ensured without any significant change in the system volume due to the action of the barostat. Pressure and temperature are controlled by the Nosé-Hoover thermostat and barostat with relaxation parameters 0.5 and 0.5 , and the time step in our NPT simulations is 2 fs.

The evolution of the metastable system is monitored by storing the positions of the atoms every 1000 time steps. As the metastable system is free to develop larger clusters over time, a cluster monitoring procedure was developed to calculate the number of clusters and their sizes in each of the stored configurations. The cluster search algorithm uses the Stirling criterion with $2.5 \AA \mathrm{O}-\mathrm{H}$ distance [9]. 


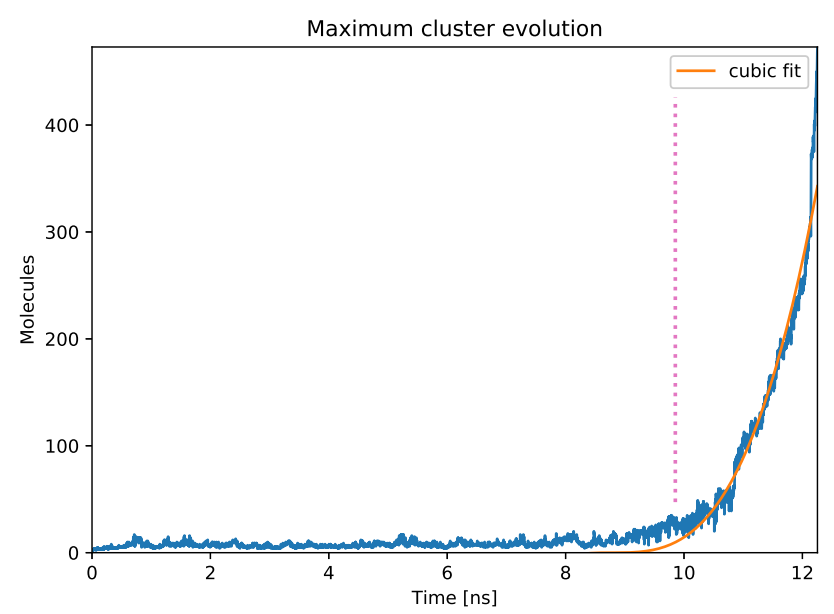

Fig. 1 Evolution of the largest cluster in NPT simulation run (500 TIP4P/2005 molecules, $425 \mathrm{~K}, 590 \mathrm{kPa}$ ). Critical cluster size according to CNT is 19.5 molecules. The vertical dotted line denotes the nucleation time.

\subsection{Growth of the liquid cluster}

The transition of the system to the growth stage is observed, when the size of the maximum cluster exceeds the critical size and progresses in its growth until the simulated system consists entirely of the liquid phase. Fig. 1 shows the evolution of the maximum cluster size in a single NPT run of our example simulation.

In Fig. 1, a clear distinction can be observed between the initial incubation stage, which lasts for about $10 \mathrm{~ns}$, and the subsequent growth stage. The transition to continuous growth of the maximum cluster marks the nucleation event. During the incubation stage, the system resides in a metastable state for a period of time and is characterized by fluctuations in the maximum cluster size, which can even exceed the critical cluster size slightly, but they break up. Also during the incubation stage, the system fluctuates around a constant volume as can be seen in Fig. 2. The growth stage is characterized by a continuous growth of the maximum cluster in the system. During the growth stage, the maximum cluster is scavenging the gas molecules and small clusters from the system. Since the system is maintained at a constant temperature and pressure by the action of the thermostat and barostat, the transition of molecules from the vapor phase to the liquid phase manifests itself by a considerable decrease in the volume of the system, as can be seen in Fig. 2. Similarly, the density of both monomers and small clusters decreases as the system nucleates. This can be observed from the number of monomers in Fig. 3 and the number of clusters with sizes in the range of two to nine molecules in Fig. 4.

To calculate the time of nucleation $t_{n u c}$ in our simulation run, we iteratively search for the transition between the incubation stage and the growth stage as 


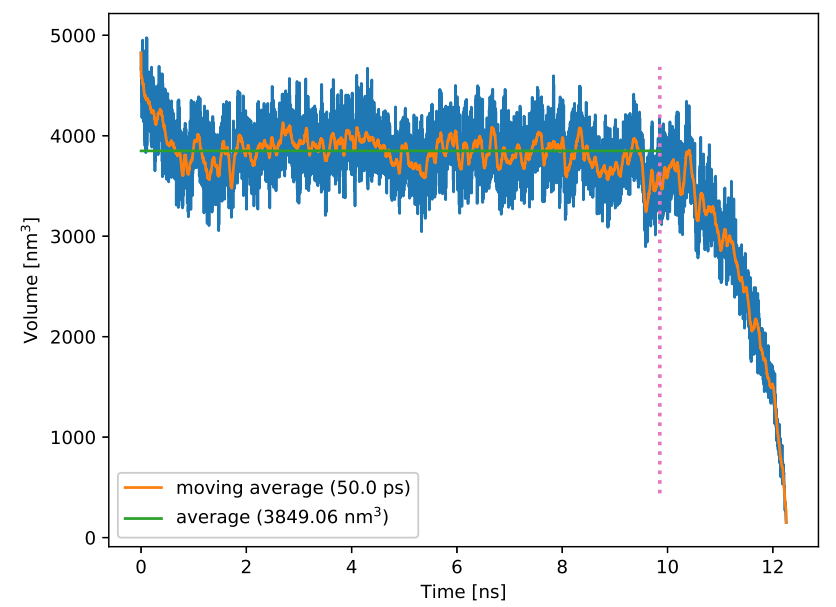

Fig. 2 Volume of the simulation box during an NPT simulation run (500 TIP4P/2005 molecules, $425 \mathrm{~K}, 590 \mathrm{kPa}$ ).

follows. First, we make a rough estimate of the nucleation time $t_{n u c, 0}$ by considering the nucleation time as the time when the maximum cluster becomes larger than $20 \%$ of the total system molecules. In the first iteration, an average of the maximum cluster sizes $n_{i n c, 0}$ for the incubation period from the simulation start to $t_{n u c, 0}$ is evaluated, and a cubic fit $c_{g r, 0}$ of the maximum cluster sizes for the growth period between $t_{n u c, 0}$ and the simulation end is evaluated as well. A second estimate for the nucleation time $t_{n u c, 1}$ is calculated as the time, at which the cubic fit of cluster growth equals the average cluster size during the incubation period $c_{g r, 0}\left(t_{n u c, 1}\right)=n_{i n c, 0}$. The new estimate of the nucleation time $t_{n u c, 1}$ is used in the second iteration to evaluate the average cluster size $n_{i n c, 1}$ during the incubation stage and the cubic growth function $c_{g r, 1}$ during the growth stage, etc. After several iterations, the search for the nucleation time is finished under the condition $\left|t_{n u c, i+1}-t_{n u c, i}\right|<0.01 \mathrm{~ns}$. The nucleation time estimated by this numerical method is shown in Figs. $1-$ 4 by the dotted vertical line at $9.8 \mathrm{~ns}$, and the final cubic fit of the maximum cluster growth is shown by the orange line in Fig. 1.

\subsubsection{Cluster growth rate}

The growth of the cluster beyond the critical cluster size is characterized by a power-law dependence of the size on time. Assuming that the impingement rate of the vapor molecules on the cluster surface is constant, the size evolution of the cluster is represented by a cubic function as described in Appendix B. This behavior can be observed in Fig. 1 (orange line).

The attachment of the vapor molecules to the growing cluster can be evaluated in the MD experiment by comparing the maximum cluster size between successive configurations stored during the numerical simulation. The instantaneous growth rate calculated in this way is plotted in Fig. 5 (blue line). 


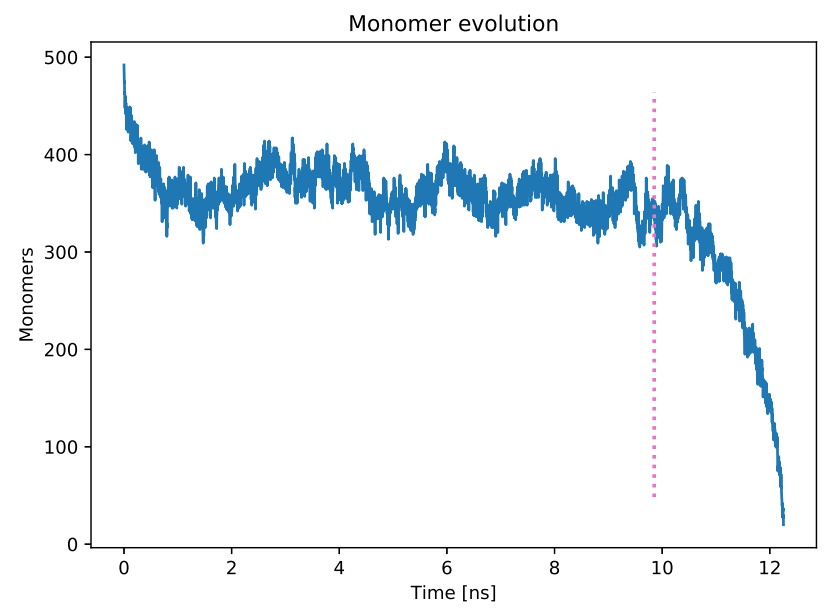

Fig. 3 Distribution of monomers during an NPT simulation run (500 TIP4P/2005 molecules, $425 \mathrm{~K}, 590 \mathrm{kPa}$ ).

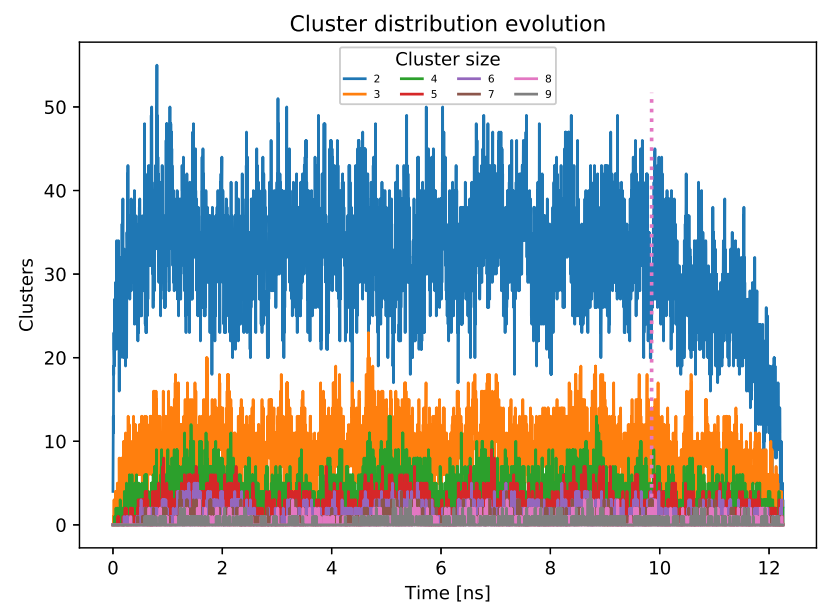

Fig. 4 Distribution of clusters of sizes $2-9$ during an NPT simulation run (500 TIP4P/2005 molecules, $425 \mathrm{~K}, 590 \mathrm{kPa}$ ).

After fitting this stochastic growth process to the cubic model according to Appendix B, a good agreement is observed between the resulting average impingement rate $\beta_{M D}$ (green dotted line) and the impingement rate model used by CNT (orange line) in Fig. 5 .

\subsubsection{Nucleation rate}

Since the nucleation event is a stochastic phenomenon, variations in the nucleation time of parallel NPT simulation runs starting from different initial 


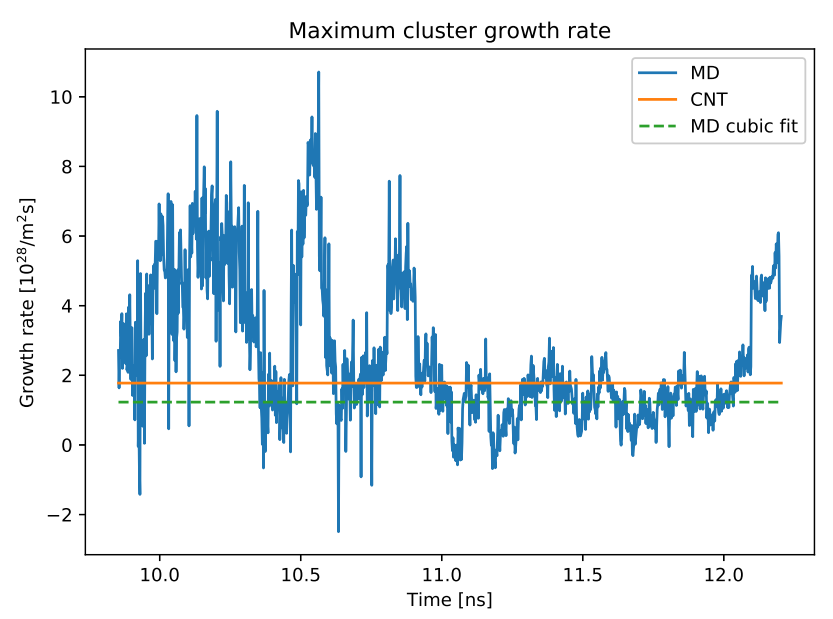

Fig. 5 Growth rate of the maximum cluster during the growth stage of an NPT simulation run (500 TIP4P/2005 molecules, $425 \mathrm{~K}, 590 \mathrm{kPa}$ ).

configurations are to be expected, even though all simulation runs have the same target pressure and temperature. For the 20 parallel simulation runs of our example system, the nucleation times shown in Fig. 6 span from a few nanoseconds to over $100 \mathrm{~ns}$. Note that such a wide range of nucleation times shows that a nucleation rate measured in a single-run MD simulation $[9,11]$ is a poor estimate of the steady-state nucleation rate.

To calculate the average nucleation time $t_{n u c}$, a log-normal distribution of nucleation times is assumed. A log-normal distribution of nucleation times corresponds to normal distribution of the critical nucleation work due to relation (A4). In our example case, the log-normal average nucleation time is $23.8 \mathrm{~ns}$, where the standard deviation (one-sigma) corresponds to the interval $(11.1,51.0) \mathrm{ns}$.

After estimating the nucleation time, the nucleation rate is evaluated as

$$
J_{M D}=\frac{1}{V_{\text {avg }} t_{n u c}}
$$

where $V_{\text {avg }}$ is the average volume of the system during the incubation stages (Fig. 2) of the set of 20 simulation runs under evaluation. A well-established incubation phase during the NPT simulation runs is essential for a reliable measurement of $V_{\text {avg }}$. The standard deviation of the average volume in the 20 simulation runs of our example case was $0.5 \%$.

The resulting nucleation rate according to Eq. (1) in our example system is $1.1 \times 10^{31} \mathrm{~m}^{-3} \mathrm{~s}^{-1}$ with standard deviation between $5.1 \times 10^{30}$ and $2.4 \times 10^{31}$. We see that the original nucleation rate estimate by CNT of $2.2 \times 10^{31} \mathrm{~m}^{-3} \mathrm{~s}^{-1}$ (Table 2) falls within the standard deviation of our MD nucleation rate measurement in this example case. 


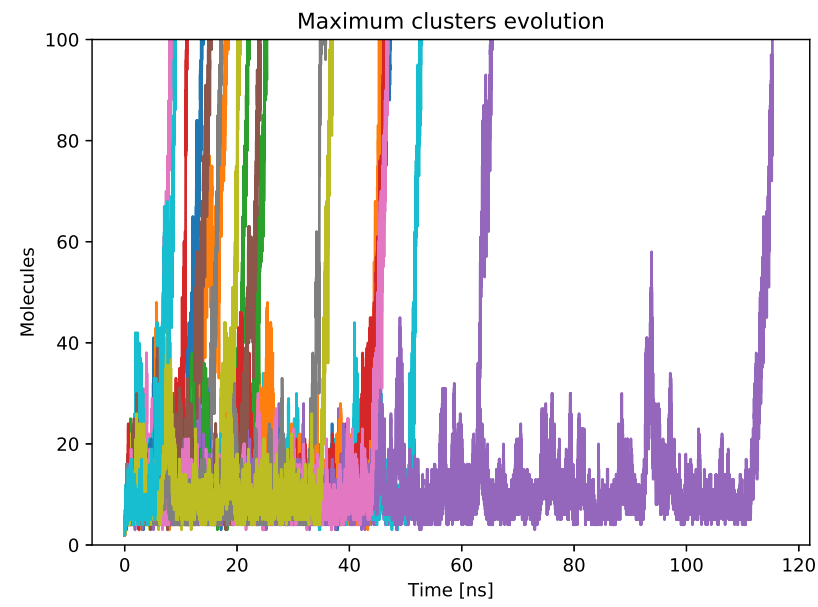

Fig. 6 Evolution of maximum clusters in a set of 20 NPT simulation runs (500 TIP4P/2005 molecules, $425 \mathrm{~K}, 590 \mathrm{kPa})$. The log-normal average nucleation time $t_{n u c}$ for this set of simulation runs is $23.8 \mathrm{~ns}$.

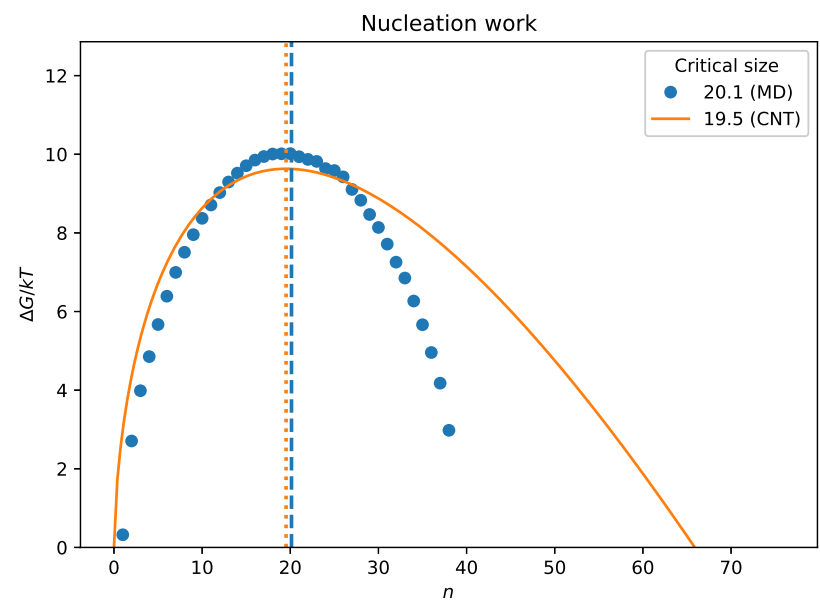

Fig. 7 Nucleation work as a function of cluster molecules calculated for a set of 20 NPT simulation runs (500 TIP4P/2005 molecules, $425 \mathrm{~K}, 590 \mathrm{kPa}$ ) (dots) vs. predicted by CNT (orange line).

\subsubsection{Nucleation work}

A standard approach to extracting the nucleation work $\Delta G_{M D}$ from the cluster size distribution is used in this work, as described in Appendix C. Fig. 7 shows the nucleation work calculated from the cluster distributions for our example system according to Eq. (C11) compared to the CNT nucleation work calculated according to Eq. (A1). 
The vertical lines in Fig. 7 represent the critical cluster sizes. For CNT (dotted orange line), the critical size is calculated according to Eq. (A3). For the set of 20 NPT simulations, the nucleation work data (blue points) are fitted to a CNT-like nucleation work function $\Delta G_{M D}(n)=p_{1} n^{2 / 3}-p_{2} n$, and the maximum of the fitted function is calculated analytically (dashed blue line).

\section{Results and discussion}

According to the above described procedure, nucleation parameters of two water models, SPC/E and TIP4P/2005, were evaluated from our MD simulations and compared to CNT predictions.

For SPC/E, nucleation simulations for temperatures in the range $300-$ $425 \mathrm{~K}$ were performed for systems of 108 molecules in order to monitor the temperature dependence of the nucleation rate. At $375 \mathrm{~K}$, additional system sizes of 500 and 2916 molecules were simulated in order to observe the pressure dependence of the nucleation rate, because larger systems at lower supersaturations allow for the simulation of lower nucleation rates.

For TIP 4P / 2005, simulations of 108 molecules at temperatures in the range $325-475 \mathrm{~K}$ were run. At $425 \mathrm{~K}$, system sizes of 500 and 2916 molecules were simulated as well.

\subsection{Nucleation rate}

The nucleation rates measured in SPC/E simulations are plotted in Fig. 8 and the corresponding data points are summarized in Table 3 together with uncertainty intervals (one-sigma in the logarithmic scale). We can see that the nucleation rates measured in numerical experiments are quite precisely reproduced by CNT. In 4 out of the total 8 cases, the CNT nucleation rates lie within the uncertainty interval of MD nucleation rate, and in 4 other cases the CNT nucleation rate differs from the MD rates by a factor less than $2.5 \times$. The worst case shows a difference of $2.14 \times 10^{31} \mathrm{~m}^{-3} \mathrm{~s}^{-1}$ CNT nucleation rate vs. $5.34 \times 10^{31} \mathrm{~m}^{-3} \mathrm{~s}^{-1}$ MD nucleation rate. These results show a very good compatibility between CNT predictions and our MD simulations, which is a situation quite different from the typical discrepancies of several orders of magnitude in the nucleation rate as reported by previous molecular dynamics studies or laboratory nucleation experiments.

At $375 \mathrm{~K}, \mathrm{SPC} / \mathrm{E}$ simulations of larger systems allowed us to evaluate the pressure dependence of the MD nucleation rate. The slope of the $375 \mathrm{~K} \mathrm{CNT}$ isotherm in Fig. 8 is again reproducing the slope of MD nucleation rate data quite accurately.

The comparison of our SPC/E results and nucleation rates reported by Angelil et al. [7] is shown in Fig. 9. Both data sets are reproduced by CNT accurately. For Angelil et al. data, we actually found a closer agreement with CNT than the authors reported. This is caused by the slightly different parametrizations of $\mathrm{SPC} / \mathrm{E}$ thermophysical properties employed in the respective $\mathrm{CNT}$ 


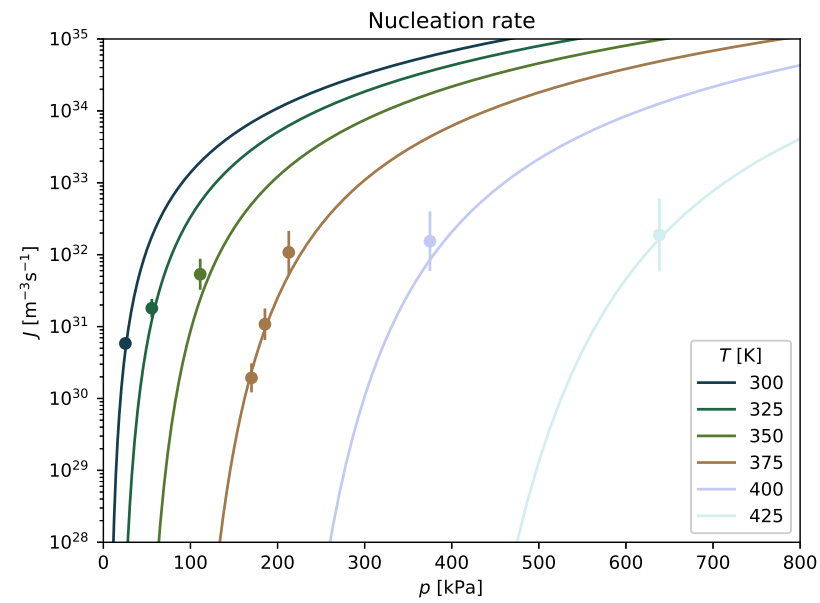

Fig. 8 Nucleation rate in SPC/E water by numerical experiments (dots) vs. nucleation rate predictions by CNT (lines).

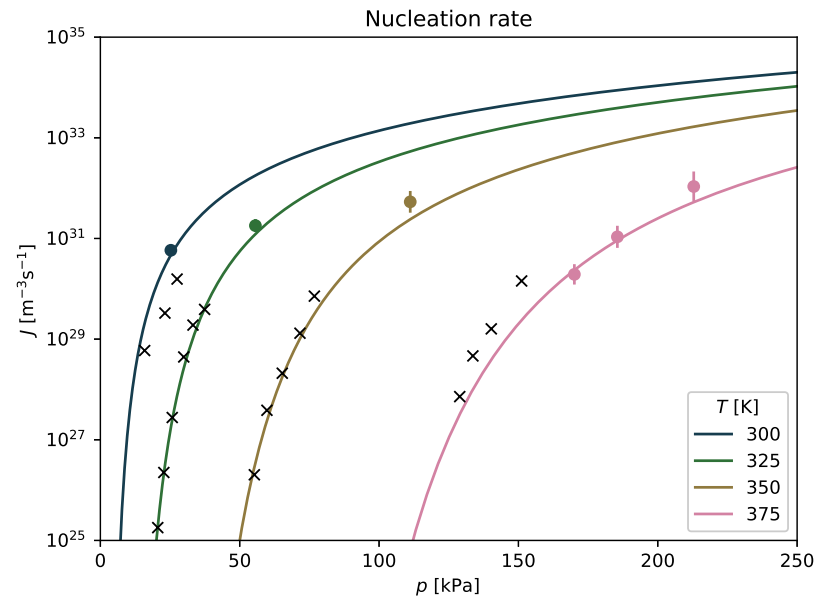

Fig. 9 Nucleation rate in SPC/E water performed in this work (dots) compared with nucleation rates measured in NVT simulations by Angelil et al. [7] (crosses) and with CNT predictions (lines).

calculations, as the accurate NIST SPC/E thermophysical properties [16] were not available at the time of Angelil et al. work.

The other SPC/E NVT nucleation rates reported in the literature (Table 1) deviate from CNT by several orders of magnitude. Therefore, the agreement between the CNT prediction and Angelil et al. NVT data might seem coincidental. However, since Angelil et al. performed large-scale molecular simulations (up to 4.1 million SPC/E molecules), the pressure during their NVT simulation runs was not decreasing substantially due to droplet formation 


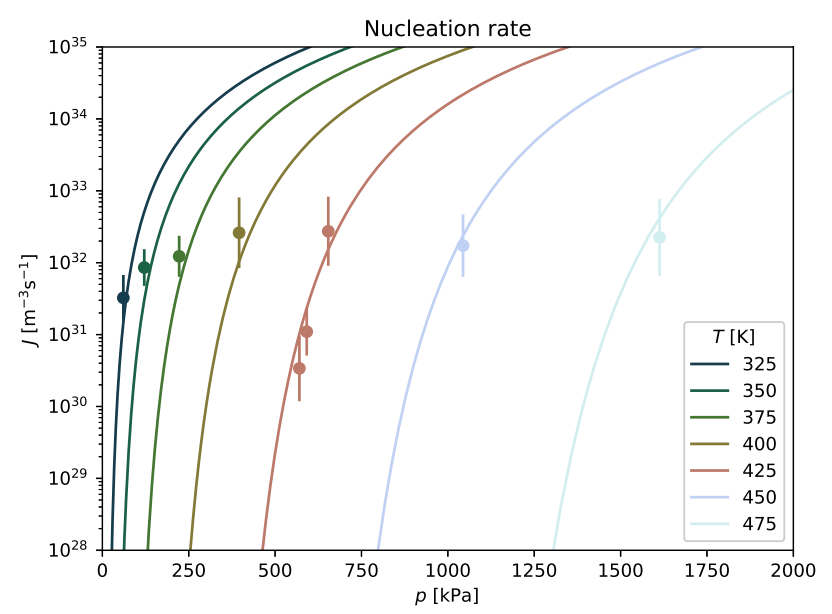

Fig. 10 Nucleation rate in TIP4P/2005 water by numerical experiments (dots) vs. nucleation rate predictions by CNT (lines).

and as such the simulated process was resembling the steady-state nucleation process also quite closely.

The nucleation rates measured in TIP $4 \mathrm{P} / 2005$ simulations are plotted in Fig. 10, and the data points are given in Table 5 . The agreement between CNT predictions and MD nucleation rates is again very good. For 5 out of the total 9 cases, the CNT nucleation rates lie within the uncertainty interval of MD nucleation rate, and in the other cases the CNT nucleation rate differs from the MD rates by a factor of less than $3.1 \times$. The worst case shows a difference of $8.56 \times 10^{31} \mathrm{~m}^{-3} \mathrm{~s}^{-1} \mathrm{CNT}$ nucleation rate vs. $2.78 \times 10^{32} \mathrm{~m}^{-3} \mathrm{~s}^{-1} \mathrm{MD}$ nucleation rate.

The level of agreement between CNT predictions and our MD measurements is basically the same for both water models. In both cases, best agreement can be observed for high temperatures, i.e. for simulations with larger critical clusters.

To justify our choice of 20 simulations as sufficient statistics for averaging the nucleation rate, or, in other words, to illustrate the precision of evaluating the nucleation rate from sets of 20 parallel nucleation simulations, nucleation rates were evaluated for 6 sets of 20 simulations at the same thermodynamic state of the parent vapor, i.e. $450 \mathrm{~K}$ and $1030 \mathrm{kPa}$, as shown in Fig. 11. The data points differ by a factor less than $2 \times$ in the absolute value of the nucleation rate, while the respective uncertainty intervals overlap to a large extent. The differences in the temperature during the incubation stages of the numerical experiment measured as an average over the set of 20 simulation runs are of the order of $0.01 \mathrm{~K}$ and the differences in pressure measured in the same way show an order of $1 \mathrm{kPa}$. 


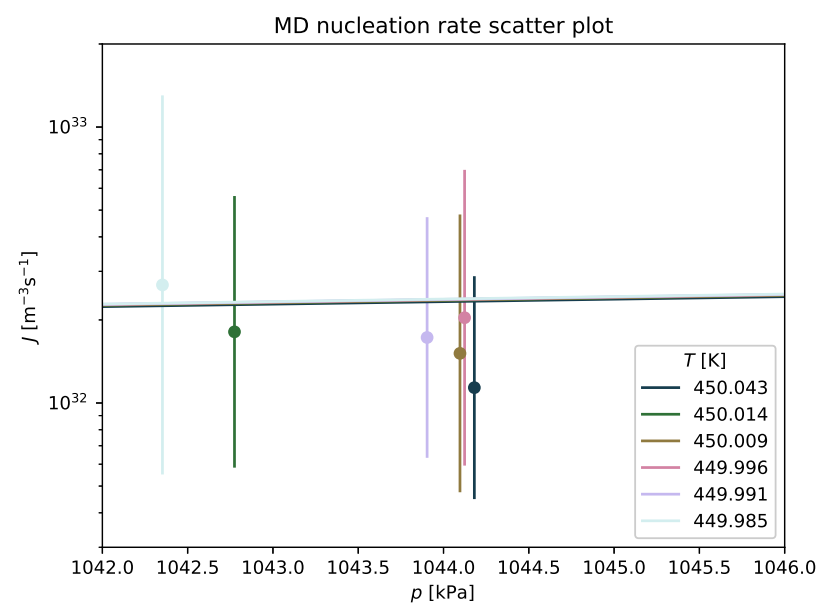

Fig. 11 Nucleation rate in TIP4P/2005 water by 6 numerical experiments each consisting of 20 simulation runs at $450 \mathrm{~K}$ and $1030 \mathrm{kPa}$.

\subsection{Impingement rate}

The impingement rate evaluated from our numerical experiments according to the procedure described in Sec. 2.4.1 is summarized in the last columns of Tables 3 and 5, for SPC/E and TIP4P/2005 model, respectively. A very good agreement with CNT impingement rates calculated according to the basic ideal-gas model (A7) is observed. For SPC/E, the difference in the worst case is by a factor of $2.6 \times$.

For TIP $4 \mathrm{P} / 2005$, the worst difference factor is $7.5 \times$ at $475 \mathrm{~K}$, which is the highest simulated temperature. It should be noted, however, that the molecular system at $475 \mathrm{~K}$ is on the edge of its ability to spawn the steady-state nucleation process properly. The critical cluster size for $475 \mathrm{~K}$ simulation runs is almost 30, while the total number of molecules in the simulation is 108 only. In such case, the size fluctuations of the largest cluster around the critical size may reach the total size of the system. In other words, the growth/decay behavior of the nucleating cluster around its critical size may feature situations, when the cluster interacts with itself, because there is not enough vapor molecules surrounding it, effectively altering the nucleation process.

\subsection{Nucleation work and critical cluster size}

Critical nucleation work and the corresponding critical cluster size have been evaluated from molecular simulations according to the procedure described in Sec. 2.4.3.

For SPC/E, the results are summarized in Table 4. The critical clusters range in size from 5.8 to 15.5 molecules in our MD experiments. Smaller critical sizes have been achieved for lower system temperatures, which reflects the way of selecting the system temperatures and pressures in order to arrive at 
Table $3 \mathrm{SPC} / \mathrm{E}$ nucleation rate $J$ and impingement rate $\beta$ predicted by CNT vs. measured in MD simulation.

\begin{tabular}{ccc|ccc|cc}
\hline $\begin{array}{c}T \\
{[\mathrm{~K}]}\end{array}$ & $\begin{array}{c}p \\
{[\mathrm{kPa}]}\end{array}$ & $n$ & $\begin{array}{c}J_{C N T} \\
{\left[\mathrm{~m}^{-3} \mathrm{~s}^{-1}\right]}\end{array}$ & $\begin{array}{c}J_{M D} \\
{\left[\mathrm{~m}^{-3} \mathrm{~s}^{-1}\right]}\end{array}$ & $\begin{array}{c}\Delta J_{M D} \\
{\left[\mathrm{~m}^{-3} \mathrm{~s}^{-1}\right]}\end{array}$ & $\begin{array}{c}\beta_{C N T} \\
{\left[\mathrm{~m}^{-2} \mathrm{~s}^{-1}\right]}\end{array}$ & $\begin{array}{c}\beta_{M D} \\
{\left[\mathrm{~m}^{-2} \mathrm{~s}^{-1}\right]}\end{array}$ \\
\hline 300 & 25 & 108 & $4.36 \times 10^{30}$ & $5.83 \times 10^{30}$ & $\left(4.80 \times 10^{30}, 7.07 \times 10^{30}\right)$ & $8.96 \times 10^{26}$ & $1.34 \times 10^{27}$ \\
325 & 55 & 108 & $1.12 \times 10^{31}$ & $1.80 \times 10^{31}$ & $\left(1.34 \times 10^{31}, 2.42 \times 10^{31}\right)$ & $1.89 \times 10^{27}$ & $3.03 \times 10^{27}$ \\
350 & 110 & 108 & $2.14 \times 10^{31}$ & $5.34 \times 10^{31}$ & $\left(3.25 \times 10^{31}, 8.78 \times 10^{31}\right)$ & $3.65 \times 10^{27}$ & $5.04 \times 10^{27}$ \\
375 & 210 & 108 & $4.54 \times 10^{31}$ & $1.08 \times 10^{32}$ & $\left(5.42 \times 10^{31}, 2.14 \times 10^{32}\right)$ & $6.73 \times 10^{27}$ & $8.73 \times 10^{27}$ \\
375 & 185 & 500 & $8.89 \times 10^{30}$ & $1.08 \times 10^{31}$ & $\left(6.50 \times 10^{30}, 1.79 \times 10^{31}\right)$ & $5.93 \times 10^{27}$ & $5.04 \times 10^{27}$ \\
375 & 170 & 2916 & $2.37 \times 10^{30}$ & $1.94 \times 10^{30}$ & $\left(1.21 \times 10^{30}, 3.08 \times 10^{30}\right)$ & $5.45 \times 10^{27}$ & $2.08 \times 10^{27}$ \\
400 & 370 & 108 & $6.97 \times 10^{31}$ & $1.54 \times 10^{32}$ & $\left(5.92 \times 10^{31}, 4.00 \times 10^{32}\right)$ & $1.15 \times 10^{28}$ & $1.08 \times 10^{28}$ \\
425 & 630 & 108 & $1.29 \times 10^{32}$ & $1.88 \times 10^{32}$ & $\left(5.86 \times 10^{31}, 6.06 \times 10^{32}\right)$ & $1.90 \times 10^{28}$ & $2.09 \times 10^{28}$ \\
\hline
\end{tabular}

Table 4 SPC/E critical cluster size $n^{\star}$ and critical nucleation work $\Delta G^{\star}$ predicted by CNT vs. measured in MD simulation.

\begin{tabular}{ccc|cc|cc}
\hline $\begin{array}{c}T \\
{[\mathrm{~K}]}\end{array}$ & $\begin{array}{c}p \\
{[\mathrm{kPa}]}\end{array}$ & $n$ & $n_{C N T}^{*}$ & $n_{M D}^{*}$ & $\frac{W_{C N T}^{*}}{k T}$ & $\frac{W_{M D}^{*}}{k T}$ \\
\hline 300 & 25 & 108 & 3.7 & 5.8 & 5.7 & 6.1 \\
325 & 55 & 108 & 5.1 & 8.5 & 6.1 & 6.9 \\
350 & 110 & 108 & 7.1 & 8.8 & 6.7 & 6.9 \\
375 & 210 & 108 & 9.5 & 11.0 & 7.1 & 7.3 \\
375 & 185 & 500 & 12.4 & 15.1 & 8.5 & 8.8 \\
375 & 170 & 2916 & 15.0 & 14.8 & 9.6 & 9.6 \\
400 & 370 & 108 & 13.2 & 12.6 & 7.6 & 7.7 \\
425 & 630 & 108 & 17.6 & 15.5 & 7.9 & 8.7 \\
\hline
\end{tabular}

Table 5 TIP4P/2005 nucleation rate $J$ and impingement rate $\beta$ predicted by CNT vs. measured in MD simulation.

\begin{tabular}{ccc|ccc|cc}
\hline $\begin{array}{c}T \\
{[\mathrm{~K}]}\end{array}$ & $\begin{array}{c}p \\
{[\mathrm{kPa}]}\end{array}$ & $n$ & $\begin{array}{c}J_{C N T} \\
{\left[\mathrm{~m}^{-3} \mathrm{~s}^{-1}\right]}\end{array}$ & $\begin{array}{c}J_{M D} \\
{\left[\mathrm{~m}^{-3} \mathrm{~s}^{-1}\right]}\end{array}$ & $\begin{array}{c}\Delta J_{M D} \\
{\left[\mathrm{~m}^{-3} \mathrm{~s}^{-1}\right]}\end{array}$ & $\begin{array}{c}\beta_{C N T} \\
{\left[\mathrm{~m}^{-2} \mathrm{~s}^{-1}\right]}\end{array}$ & $\begin{array}{c}\beta_{M D} \\
{\left[\mathrm{~m}^{-2} \mathrm{~s}^{-1}\right]}\end{array}$ \\
\hline 325 & 60 & 108 & $1.37 \times 10^{31}$ & $3.25 \times 10^{31}$ & $\left(1.56 \times 10^{31}, 6.74 \times 10^{31}\right)$ & $2.07 \times 10^{27}$ & $2.99 \times 10^{27}$ \\
350 & 120 & 108 & $2.78 \times 10^{31}$ & $8.56 \times 10^{31}$ & $\left(4.76 \times 10^{31}, 1.54 \times 10^{32}\right)$ & $3.98 \times 10^{27}$ & $4.18 \times 10^{27}$ \\
375 & 220 & 108 & $4.45 \times 10^{31}$ & $1.23 \times 10^{32}$ & $\left(6.36 \times 10^{31}, 2.37 \times 10^{32}\right)$ & $7.05 \times 10^{27}$ & $6.66 \times 10^{27}$ \\
400 & 390 & 108 & $8.55 \times 10^{31}$ & $2.61 \times 10^{32}$ & $\left(8.42 \times 10^{31}, 8.08 \times 10^{32}\right)$ & $1.21 \times 10^{28}$ & $1.31 \times 10^{28}$ \\
425 & 645 & 108 & $1.20 \times 10^{32}$ & $2.74 \times 10^{32}$ & $\left(9.03 \times 10^{31}, 8.29 \times 10^{32}\right)$ & $1.94 \times 10^{28}$ & $2.31 \times 10^{28}$ \\
425 & 590 & 500 & $2.18 \times 10^{31}$ & $1.10 \times 10^{31}$ & $\left(5.10 \times 10^{30}, 2.36 \times 10^{31}\right)$ & $1.78 \times 10^{28}$ & $7.79 \times 10^{27}$ \\
425 & 570 & 2916 & $9.98 \times 10^{30}$ & $3.39 \times 10^{30}$ & $\left(1.18 \times 10^{30}, 9.71 \times 10^{30}\right)$ & $1.72 \times 10^{28}$ & $3.97 \times 10^{27}$ \\
450 & 1030 & 108 & $1.76 \times 10^{32}$ & $1.73 \times 10^{32}$ & $\left(6.33 \times 10^{31}, 4.71 \times 10^{32}\right)$ & $3.01 \times 10^{28}$ & $5.00 \times 10^{28}$ \\
475 & 1590 & 108 & $2.75 \times 10^{32}$ & $2.25 \times 10^{32}$ & $\left(6.51 \times 10^{31}, 7.76 \times 10^{32}\right)$ & $4.53 \times 10^{28}$ & $3.38 \times 10^{29}$ \\
\hline
\end{tabular}

Table 6 TIP4P $/ 2005$ critical cluster size $n^{\star}$ and critical nucleation work $\Delta G^{\star}$ predicted by CNT vs. measured in MD simulation.

\begin{tabular}{ccc|cc|cc}
\hline $\begin{array}{c}T \\
{[\mathrm{~K}]}\end{array}$ & $\begin{array}{c}p \\
{[\mathrm{kPa}]}\end{array}$ & $n$ & $n_{C N T}^{*}$ & $n_{M D}^{*}$ & $\frac{W_{C N T}^{*}}{k T}$ & $\frac{W_{M D}^{*}}{k T}$ \\
\hline 325 & 60 & 108 & 4.4 & 6.0 & 6.2 & 6.4 \\
350 & 120 & 108 & 6.0 & 7.0 & 6.7 & 6.4 \\
375 & 220 & 108 & 8.3 & 8.2 & 7.2 & 6.9 \\
400 & 390 & 108 & 10.9 & 11.7 & 7.6 & 7.4 \\
425 & 645 & 108 & 15.1 & 14.0 & 8.1 & 8.3 \\
425 & 590 & 500 & 19.5 & 20.0 & 9.6 & 10.4 \\
425 & 570 & 2916 & 21.7 & 20.5 & 10.3 & 10.7 \\
450 & 1030 & 108 & 20.5 & 19.4 & 8.5 & 10.2 \\
475 & 1590 & 108 & 27.8 & 33.2 & 8.8 & 12.1 \\
\hline
\end{tabular}


sufficiently long nucleation times in the respective simulations. Slight underprediction (of up to $40 \%$ ) of the critical cluster size by CNT can be observed for temperatures below $375 \mathrm{~K}$ and for smaller critical clusters. For higher temperatures, the critical cluster size is being slightly overpredicted (less than 14 \%) by CNT. The critical nucleation work $\Delta G_{M D}^{\star}$ is reproduced by CNT quite accurately over the whole range of $\mathrm{SPC} / \mathrm{E}$ simulations.

For TIP4P $/ 2005$, critical cluster parameters are summarized in Table 6 , showing a range of critical clusters sizes from 6.0 to 33.2 molecules. We can observe a similar level of agreement between numerical results and CNT predictions as in the case of SPC/E simulations; the differences between theoretical critical cluster size and MD experiment is less than 2 molecules. The critical nucleation work is also reproduced by CNT quite accurately over the range of simulated TIP $4 \mathrm{P} / 2005$ systems. A relatively larger deviation in both nucleation work and cluster size can be observed only in the $475 \mathrm{~K}$ case, which may represent a system in which the nucleation process deviates from the desired steady-state scenario as already mentioned above.

\section{Conclusion}

Droplet nucleation processes in water vapor were simulated using molecular dynamics (MD). Two molecular models for water were selected, SPC/E and TIP4P/2005, which have been most commonly used for nucleation simulations by other authors to date.

Nucleation rates were measured directly in the molecular simulations by observing the occurrence of the supercritical cluster. The agreement between the MD simulations and the predictions of classical nucleation theory (CNT) was found to be approximatelly in the range of standard deviation of the MD nucleation rate. A similar level of agreement was found between the impingement rate measured for the growing, just-nucleated cluster and the impingement rate calculated using the simple, ideal-gas-based model adopted by CNT. Two other nucleation parameters, i.e. the critical nucleation work and the critical cluster size, were derived from the MD cluster distributions according to classical theoretical relations between the steady-state cluster distribution and the constrained-equilibrium cluster distribution. These two nucleation parameters agree with the CNT predictions with deviations in the low double-digit percentage range, even for critical cluster sizes in the single-digit range.

Compared to the MD simulations of water nucleation previously presented in the literature, our MD simulations are distinguished by their adherence to the constant temperature and constant pressure assumptions of the CNT model. This cannot be emphasized enough; the CNT model predicts a steadystate nucleation rate and as such it has been derived assuming constant temperature and constant pressure of the parent phase during the nucleation process. Therefore, attempts to reproduce nucleation rates using CNT for processes other than NPT are bound to be inaccurate. The degree of inaccuracy of 
such attempts will be proportional to the degree of deviation of the simulated nucleation process from the steady-state nucleation case.

\section{Appendix A Classical nucleation theory overview}

The following Eqs. (A1) - (A7) summarize the formulas of the classical nucleation theory (CNT) for the case of unary homogeneous nucleation of droplets. A thorough derivation of the formulas governing the nucleation parameters can be found in nucleation textbooks, e.g. Kalikmanov [17]. The nucleation parameters reflect the thermophysical state of the nucleating parent phase characterized by pressure $p$ and temperature $T$. Within the CNT model, these two parameters are assumed constant during the nucleation process, and the required thermophysical properties of water are being evaluated at the nucleation pressure and temperature.

The nucleation work $\Delta G[\mathrm{~J}]$ within $\mathrm{CNT}$ is a function of the cluster size given by the number of molecules $n$

$$
\Delta G(n)=\sigma \sqrt[3]{36 \pi\left(v_{l} n\right)^{2}}-n k T \ln \frac{p}{p_{\text {sat }}}
$$

where $v_{l}\left[\mathrm{~m}^{-3}\right]$ is molecular volume of water molecules in the liquid phase, $p_{\text {sat }}$ $[\mathrm{Pa}]$ is the saturation pressure of water, and $\sigma[\mathrm{N} / \mathrm{m}]$ is the surface tension.

The energy barrier to nucleation denoting the maximum of the nucleation work (A1), i.e. the critical nucleation work $\Delta G^{\star}$, takes the form

$$
\Delta G^{\star}=\frac{16 \pi}{3} \frac{\sigma^{3}}{\left(k T \ln \frac{p}{p_{s a t}}\right)^{2}}
$$

The critical cluster size, conveniently expressed as the number of molecules in the critical cluster $n^{\star}$, is calculated as

$$
n^{\star}=36 \pi v_{l}^{2}\left(\frac{2}{3} \frac{\sigma}{k T \ln \frac{p}{p_{s a t}}}\right)^{3}
$$

The nucleation rate $J\left[\mathrm{~m}^{-3} \mathrm{~s}^{-1}\right]$, representing the number of droplets crossing the critical size and growing to macroscopic proportions, is given by

$$
J=J_{0} \exp \left(-\frac{\Delta G^{\star}}{k T}\right)
$$

where the pre-exponential factor $J_{0}\left[\mathrm{~m}^{-3} \mathrm{~s}^{-1}\right]$ is evaluated as

$$
J_{0}=Z \beta A^{\star} \rho_{v}
$$


which is a product of the non-equilibrium (Zeldovich) factor $Z$, impingement rate of monomers onto the cluster surface $\beta\left[\mathrm{m}^{-2} \mathrm{~s}^{-1}\right]$, surface area of the critical cluster $A^{\star}\left[\mathrm{m}^{2}\right]$, and water vapor number density $\rho_{v}\left[\mathrm{~m}^{-3}\right]$, which is calculated as a product of supersaturation ratio $p / p_{\text {sat }}$ and saturation vapor density $\rho_{\text {sat }, v}(T)\left[\mathrm{m}^{-3}\right]$.

The Zeldovich factor in Eq. (A5) takes the form

$$
Z=\frac{\sqrt[6]{36}}{3} \sqrt[3]{\frac{v_{l}}{\pi}} \sqrt{\frac{\sigma}{k T}}\left(n^{\star}\right)^{-2 / 3}
$$

and the impingement rate $\beta\left[\mathrm{m}^{-2} \mathrm{~s}^{-1}\right]$ is calculated as

$$
\beta=\frac{p}{\sqrt{2 \pi m_{1} k T}}
$$

To sum up, CNT allows us to predict a steady-state nucleation rate in metastable water vapor, which is a kinetic property of the macroscopic parent system at a pressure $p$ and temperature $T$. The CNT relations utilize the following four thermophysical properties of macroscopic water substance:

- surface tension $\sigma(T)$

- saturation pressure $p_{\text {sat }}(T)$

- saturated vapor number density $\rho_{\text {sat }, v}(T)$

- liquid number density $\rho_{l}(T)$

The above thermophysical properties of the SPC/E and TIP4P/2005 water models are summarized in Appendix D and E, respectively.

\section{Appendix B Impingement rate evaluation from cluster growth data}

The procedure to deduce the average impingement rate of vapor molecules attaching onto the cluster surface from molecular simulation data is based on the calculation of instantaneous growth and decay of the maximum cluster during the cluster growth stage. Assuming a constant impingement rate $\beta_{M D}$ $\left[\mathrm{m}^{-2} \mathrm{~s}^{-1}\right]$ and a single supercritical growing cluster, the cluster growth can be described as a change of the number of cluster molecules $n$ over time $t$ in the following manner

$$
\frac{d n}{d t}=\sqrt[3]{36 \pi\left(v_{l} n\right)^{2}} \beta_{M D}
$$

where the right hand side of Eq. (B8) represents the cluster surface area multiplied by the impingement rate. Differential equation (B8) can be readily integrated and its solution takes a simple form

$$
n=C_{0}\left(t-t_{0}\right)^{3}
$$

Finally, the parameters $C_{0}$ and $t_{0}$ can be evaluated by fitting the cubic function (B9) to the cluster growth data from MD simulation, i.e. number of 
cluster molecules vs. simulation time (as shown in Fig. 5, blue line), during the growth stage of the simulation run. The fitted value of $C_{0}$ is then related to the impingement rate $\beta_{M D}$ as

$$
\beta_{M D}=\frac{C_{0}}{\sqrt[3]{36 \pi v_{l}^{2}}}
$$

\section{Appendix C Nucleation work evaluation from cluster distribution data}

The procedure to evaluate the nucleation work $\Delta G_{M D}$ as a function of cluster size $n$ from cluster size distribution has been described in previous MD nucleation studies of water nucleation[3, 9, 12]. In this work, the cluster size distribution is calculated from saved configurations of all simulation runs in the MD simulations set, which in our case consisted of 20 simulation runs. For cluster size $n$, the nucleation work is a function constrained-equilibrium distribution of clusters

$$
\frac{\Delta G_{M D}(n)}{k T}=-\ln \frac{\rho_{e q}(n)}{\rho_{e q}(1)}
$$

where the constrained-equilibrium distribution $\rho_{e q}$ is related to the steadystate distribution $\rho$ through a recursive relation [17]

$$
J_{M D} \sum_{m=1}^{n-1} \frac{1}{\beta_{M D} A(m) \rho_{e q}(m)}=\frac{\rho(1)}{\rho_{e q}(1)}-\frac{\rho(n)}{\rho_{e q}(n)}
$$

The steady-state distribution $\rho(n)$ is the actual cluster distribution observed in MD simulation and is therefore calculated

$$
\rho(n)=\frac{1}{20} \sum_{i=1}^{20} \frac{1}{C_{i}} \sum_{j=1}^{C_{i}} \frac{c_{i j}(n)}{V_{M D, i j}}
$$

where $i$ denotes the simulation run and $j$ denotes the $j$-th configuration within run $i$ out of its total $C_{i}$ saved configurations. Further, $c_{i j}(n)$ is the number of clusters of size $n$ found in configuration $j$ of run $i$, and $V_{M D, i j}$ is the volume of the simulation cell in configuration $j$ of run $i$.

Note that to solve Eq. (C12) for $\rho_{e q}(n)$, two of the nucleation parameters, $J_{M D}$ and $\beta_{M D}$, need to be known in advance. Indeed this is the case, since both parameters are measured in our MD simulations independently. However, any errors in measuring $J_{M D}$ or $\beta_{M D}$ will inevitably lower the accuracy of evaluating $\Delta G_{M D}$. 


\section{Appendix D Thermophysical properties of SPC/E water}

Parametrizations of thermophysical properties of $\mathrm{SPC} / \mathrm{E}$ water valid for the temperature range $300-425 \mathrm{~K}$ are summarized below.

Surface tension $\sigma[\mathrm{N} / \mathrm{m}]$ was parametrized by Vega and Miguel[18] as

$$
\sigma(T)=s_{1}\left(1-\frac{T}{T_{c}}\right)^{11 / 9}\left(1-s_{2}\left(1-\frac{T}{T_{c}}\right)\right)
$$

where $s_{1}=0.20532, s_{2}=0.6132, T_{c}=625.7$.

Saturation pressure $p_{\text {sat }}[\mathrm{Pa}]$ data reported by NIST[16] have been fitted to an Antoine-type of function

$$
p_{\text {sat }}(T)=10^{5} \exp \left(p_{1}+\frac{p_{2}}{T+p_{3}}\right)
$$

where $p_{1}=12.74080, p_{2}=-4712.723, p_{3}=-25.67480$.

Saturated vapor density $\rho_{\text {sat }, v}\left[\mathrm{~m}^{-3}\right]$ data reported by NIST[16] have been fitted to a polynomial function in exponent as

$$
\rho_{\text {sat }, v}(T)=\exp \left(\sum_{i=0}^{4} \frac{a_{i}}{T^{i}}\right)
$$

where $a_{0}=2.855 \times 10^{1}, a_{1}=-2.897 \times 10^{4}, a_{2}=1.304 \times 10^{7}, a_{3}=-3.116 \times 10^{9}$, $a_{4}=2.722 \times 10^{11}$.

Liquid density $\rho_{l}\left[\mathrm{~m}^{-3}\right]$ data reported by NIST[16] have been fitted to a polynomial function

$$
\rho_{l}(T)=\sum_{i=0}^{4} \frac{b_{i}}{T^{i}}
$$

where $b_{0}=-4.686 \times 10^{3}, b_{1}=7.169 \times 10^{6}, b_{2}=-3.533 \times 10^{9}, b_{3}=7.954 \times 10^{11}$, $b_{4}=-6.815 \times 10^{13}$.

\section{Appendix E Thermophysical properties of TIP4P/2005 water}

Parametrizations of thermophysical properties of TIP4P/2005 water valid for the temperature range $325-475 \mathrm{~K}$ follow below.

Surface tension $\sigma[\mathrm{N} / \mathrm{m}]$ was parametrized by Vega and Miguel[18] as Eq. (D14), where $s_{1}=0.22786, s_{2}=0.6413, T_{c}=641.4$.

Saturation pressure $p_{\text {sat }}[\mathrm{Pa}]$ data reported by Vega et al. [19] have been fitted to an Antoine-type of function (D15), $p_{1}=12.46120, p_{2}=-4476.552$, $p_{3}=-41.49840$. 
Saturated vapor density $\rho_{\text {sat }, v}\left[\mathrm{~m}^{-3}\right]$ data reported by Vega et al. [19] have been fitted to function Eq. (D16), where $a_{0}=3.578 \times 10^{1}, a_{1}=-3.935 \times 10^{4}$, $a_{2}=1.825 \times 10^{7}, a_{3}=-4.216 \times 10^{9}, a_{4}=3.526 \times 10^{11}$.

Liquid density $\rho_{l}\left[\mathrm{~m}^{-3}\right]$ data reported by Vega et al. [19] have been fitted to function Eq. (D17), where $b_{0}=-5.385 \times 10^{3}, b_{1}=8.130 \times 10^{6}, b_{2}=$ $-3.958 \times 10^{9}, b_{3}=8.6345 \times 10^{11}, b_{4}=-7.067 \times 10^{13}$.

\section{References}

[1] Berendsen, H.J.C., Grigera, J.R., Straatsma, T.P.: The missing term in effective pair potentials. J. Phys. Chem. 91(24), 6269-6271 (1987). https: //doi.org/10.1021/j100308a038

[2] Abascal, J.L.F., Vega, C.: A general purpose model for the condensed phases of water: TIP4P/2005. J. Chem. Phys. 123(23), 234505 (2005). https://doi.org/10.1063/1.2121687

[3] Matsubara, H., Koishi, T., Ebisuzaki, T., Yasuoka, K.: Extended study of molecular dynamics simulation of homogeneous vapor-liquid nucleation of water. J. Chem. Phys. 127(21), 214507 (2007). https://doi.org/10.1063/ 1.2803899

[4] Sepehri, A., Amjad-Iranagh, S., Golzar, K., Modarress, H.: Homogeneous and heterogeneous nucleation of water vapor: A comparison using molecular dynamics simulation. Chem. Phys. 423, 135-141 (2013). https: //doi.org/10.1016/j.chemphys.2013.07.005

[5] Zhang, C., Wang, Y., Liu, Y., Yang, Y.: A molecular dynamics study of water vapor nucleation in the presence of ions. Chem. Eng. Sci. 137, 308-319 (2015). https://doi.org/10.1016/j.ces.2015.06.006

[6] Tanaka, K.K., Kawano, A., Tanaka, H.: Molecular dynamics simulations of the nucleation of water: Determining the sticking probability and formation energy of a cluster. J. Chem. Phys. 140(11), 114302 (2014). https://doi.org/10.1063/1.4867909

[7] Angélil, R., Diemand, J., Tanaka, K.K., Tanaka, H.: Homogeneous SPC/E water nucleation in large molecular dynamics simulations. J. Chem. Phys. 143(6), 064507 (2015) https://arxiv.org/abs/1507.07335. https: //doi.org/10.1063/1.4928055

[8] Pérez, A., Rubio, A.: A molecular dynamics study of water nucleation using the TIP4P/2005 model. J. Chem. Phys. 135(24), 244505 (2011). https://doi.org/10.1063/1.3672063

[9] Duška, M., Němec, T., Hrubý, J., Vinš, V., Planková, B.: Molecular 
dynamics simulation of vapour-liquid nucleation of water with constant energy. In: Dančová, P., Vít, T. (eds.) EPJ Web Conf., vol. 92, p. 02013 (2015). https://doi.org/10.1051/epjconf/20159202013. http:// www.epj-conferences.org/10.1051/epjconf/20159202013

[10] Dumitrescu, L.R., Smeulders, D.M.J., Dam, J.A.M., Gaastra-Nedea, S.V.: Homogeneous nucleation of water in argon. Nucleation rate computation from molecular simulations of TIP4P and TIP4P/2005 water model. J. Chem. Phys. 146(8), 084309 (2017). https://doi.org/10.1063/1.4975623

[11] Hrubý, J., Duška, M., Němec, T., Kolovratník, M.: Nucleation rates of droplets in supersaturated steam and water vapour-carrier gas mixtures between 200 and 450 K. Proc. Inst. Mech. Eng. Part A J. Power Energy 232(5), 536-549 (2018). https://doi.org/10.1177/0957650918770939

[12] Yasuoka, K., Matsumoto, M.: Molecular dynamics of homogeneous nucleation in the vapor phase. II. Water. J. Chem. Phys. 109(19), 8463-8470 (1998). https://doi.org/10.1063/1.477510

[13] Wyslouzil, B.E., Wölk, J.: Overview: Homogeneous nucleation from the vapor phase-The experimental science. J. Chem. Phys. 145(21), 211702 (2016). https://doi.org/10.1063/1.4962283

[14] Wölk, J., Strey, R., Heath, C.H., Wyslouzil, B.E.: Empirical function for homogeneous water nucleation rates. J. Chem. Phys. 117(10), 4954-4960 (2002). https://doi.org/10.1063/1.1498465

[15] Todorov, I.T., Smith, W., Trachenko, K., Dove, M.T.: DL_POLY_3: new dimensions in molecular dynamics simulations via massive parallelism. J. Mater. Chem. 16(20), 1911 (2006). https://doi.org/10.1039/b517931a

[16] www.nist.gov: SAT-TMMC: Liquid-Vapor coexistence properties - $\mathrm{SPC} / \mathrm{E} \quad$ Water (LRC) (2018). https://www.nist.gov/mml/csd/informatics/ sat-tmmc-liquid-vapor-coexistence-properties-spce-water-lrc

[17] Kalikmanov, V.I.: Nucleation Theory. Lecture Notes in Physics, vol. 860. Springer, Dordrecht (2013). https://doi.org/10.1007/978-90-481-3643-8. http://link.springer.com/10.1007/978-90-481-3643-8

[18] Vega, C., de Miguel, E.: Surface tension of the most popular models of water by using the test-area simulation method. J. Chem. Phys. 126(15), 154707 (2007). https://doi.org/10.1063/1.2715577

[19] Vega, C., Abascal, J.L.F., Nezbeda, I.: Vapor-liquid equilibria from the triple point up to the critical point for the new generation of TIP4P-like models: TIP4P/Ew, TIP4P/2005, and TIP4P/ice. J. Chem. Phys. 125(3), 
034503 (2006). https://doi.org/10.1063/1.2215612

\section{Statements and Declarations}

Funding. This work was supported by the Institute of Thermomechanics (RVO:61388998) and by the Czech Ministry of Education, Youth and Sports under project no. LTV19017. Access to computing and storage facilities owned by parties and projects contributing to the National Grid Infrastructure MetaCentrum provided under the programme "Projects of Large Research, Development, and Innovations Infrastructures" (CESNET LM2015042) is also appreciated ( $\sim 300$ thousand CPU hours).

Competing Interests. The author has no relevant financial or non-financial interests to disclose.

Author Contributions. The author confirms sole responsibility for the following: study conception and design, realization of numerical experiments, data collection, analysis and interpretation of results, and manuscript preparation. The author thanks Michal Duška for sharing his cluster evaluation scripts.

Data Availability. The data that support the findings of this work are available from the corresponding author upon request. 